\title{
Emerging regulation and functions of autophagy
}

\author{
Patricia Boya, Fulvio Reggiori and Patrice Codogno
}

\begin{abstract}
Autophagy maintains cell, tissue and organism homeostasis through degradation. Complex post-translational modulation of the Atg (autophagy-related) proteins adds additional entry points for crosstalk with other cellular processes and helps define cell-type-specific regulations of autophagy. Beyond the simplistic view of a process exclusively dedicated to the turnover of cellular components, recent data have uncovered unexpected functions for autophagy and the autophagy-related genes, such as regulation of metabolism, membrane transport and modulation of host defenses - indicating the novel frontiers lying ahead.
\end{abstract}

The word autophagy, derived from the Greek word for "self-eating", refers to the catabolic processes through which the cell turns over its own constituents ${ }^{1}$. The proteasome is also involved in cellular degradation, but autophagy refers only to those pathways that lead to the elimination of cytoplasmic components by delivering them into mammalian lysosomes or plant and yeast vacuoles. Autophagy is often labelled as degradative, but it is more accurate to describe it as a recycling pathway to highlight its important contribution to cell physiology. Metabolites generated in the lysosomes or vacuoles as a result of autophagy are reused either as sources of energy or building blocks for the synthesis of new macromolecules. So far, three major types of autophagy have been described: macroautophagy, microautophagy and chaperone-mediated autophagy (CMA) (Fig. 1). This Review will focus on macroautophagy, hereafter referred to as autophagy.

Autophagy is characterized by the formation of double-membrane vesicles called autophagosomes, which sequester the cytoplasmic structures targeted for destruction (Fig. 1). Following autophagy induction, the Atg proteins (Table 1) assemble at a specialized site that has been named the phagophore assembly site or the pre-autophagosomal structure (PAS) ${ }^{2}$. From here, they participate (probably together with specific SNAREs and tethering factors) in the orchestrated fusion of Golgi-, endosome- and plasma-membrane-derived membranes to form the phagophore ${ }^{3}$. The PAS has been shown to emerge from regions of the mammalian endoplasmic reticulum enriched in the phosphatidylinositol-3-phosphate (PtdIns3P)-binding protein DFCP1, which have been named omegasomes ${ }^{4}$. The visualization of contact points between omegasomes and phagophores have led to the hypothesis that lipids are supplied to the nascent autophagosomes by direct transfer from the endoplasmic reticulum ${ }^{5,6}$, but other lipid sources, such as mitochondria, have also been implicated in this process ${ }^{7}$. Complete autophagosomes subsequently fuse with the lysosomes (or vacuoles in plants and yeast) to expose their content to the hydrolases in the interior of these organelles.
Autophagy has been shown to participate in physiological processes ranging from adaptation to starvation, cell differentiation and development, the degradation of aberrant structures, turnover of superfluous or damaged organelles, tumour suppression, innate and adaptive immunity, lifespan extension and cell death ${ }^{8-10}$. The identification of the Atg proteins in autophagy was a milestone in the understanding of the importance of this process ${ }^{1,9}$. Furthermore, the post-translational modifications of the Atg proteins documented so far confer great plasticity for the integrated transduction of multiple stimuli into the Atg machinery. More recently, additional functions have been assigned to autophagic structures beyond their fusion with the lysosomal compartment, as well as a plethora of non-autophagic roles for the Atg proteins.

\section{Selective types of autophagy}

Autophagy has long been considered a non-selective process for bulk degradation of either long-lived proteins or cytoplasmic components during nutrient deprivation. Numerous types of selective autophagy have been uncovered recently ${ }^{11}$. Under specific conditions, autophagosomes can thus exclusively sequester and degrade mitochondria, peroxisomes, endoplasmic reticulum, endosomes, lysosomes, lipid droplets, secretory granules, cytoplasmic aggregates, ribosomes and invading pathogens (Fig. 2a). Protein complexes in signalling cascades such as the inflammasome are also regulated through selective autophagy ${ }^{12}$. In this case, it seems that their degradation does not always require the stimulation of autophagy per se, but rather the induction of their targeting to the autophagosomes formed by basal autophagy.

Selective autophagy relies on cargo-specific autophagy receptors that facilitate cargo sequestration into autophagosomes. Autophagy receptors interact directly with the structure that needs to be specifically eliminated by autophagy, as well as the pool of the Atg8 (yeast homologue of mammalian LC3) protein family members present in the internal surface of the growing autophagosomes ${ }^{11,13}$. The latter interaction is mostly mediated

Patricia Boya is in the Department of Cellular and Molecular Biology, Centro de Investigaciones Biológicas, Consejo Superior de Investigaciones Científicas, Ramiro de Maetzu 9, 28040 Madrid, Spain. Fulvio Reggiori is in the Department of Cell Biology and Institute of Biomembranes, University Medical Centre Utrecht, Heidelberglaan 100, 3584 CX Utrecht, The Netherlands. Patrice Codogno is at INSERM U845, Necker Growth and Signaling Research Center, Université Paris Descartes, 75014 Paris, France.

e-mail: patricia.boya@csic.es; F.Reggiori@umcutrecht.nl; patrice.codogno@inserm.fr 


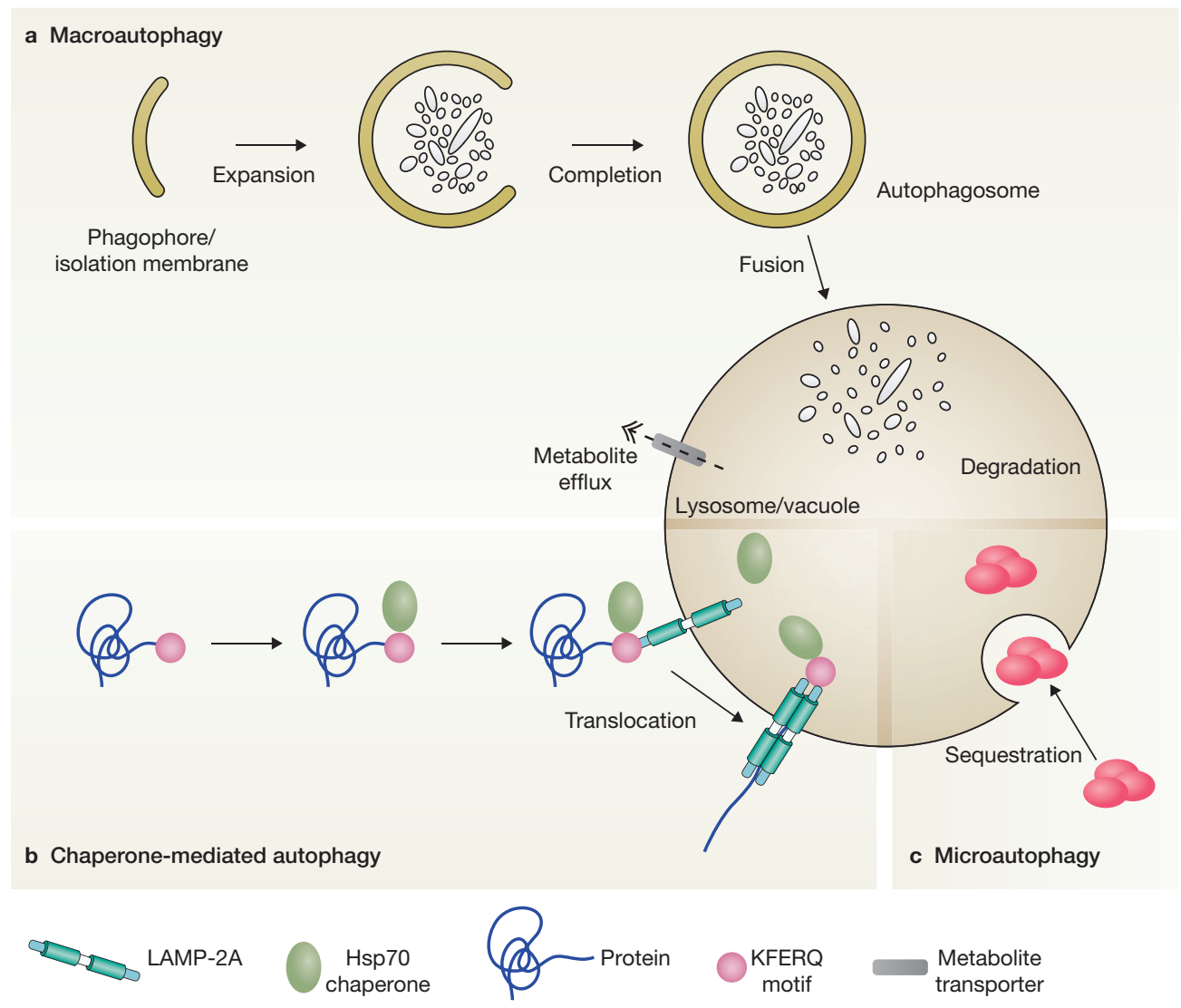

Figure 1 The different types of autophagy. (a) Macroautophagy is characterized by the sequestration of structures targeted for destruction into doublemembrane vesicles called autophagosomes. Complete autophagosomes first fuse with endosomes before finally exposing their content to the hydrolytic interior of lysosomes. The resulting metabolites are transported into the cytoplasm and used either for the synthesis of new macromolecules or as a source of energy. (b) During chaperone-mediated autophagy, proteins carrying the pentapeptide KFERQ-like sequence are recognized by the Hsc70 chaperone, which then associates with the integral lysosome membrane protein LAMP-2A, triggering its oligomerization. This event leads the translocation of the bound protein into the lysosome interior through a process that requires Hsc70. (c) Microautophagy entails the recruitment of targeted components in proximity with the lysosomal membrane, which subsequently invaginates and pinches off.

through a specific amino acid sequence present in the autophagy receptors and commonly referred to as the LC3-interacting region (LIR) or the Atg8-interacting (AIM) motif ${ }^{13,14}$.

The study of the biosynthetic transport route present in the yeast Saccharomyces cerevisiae, the cytosol-to-vacuole targeting (Cvt) pathway ${ }^{15}$, has been pivotal in understanding selective autophagy in other eukaryotic cells. This pathway mediates the delivery into the vacuole lumen of three hydrolases that are all part of a large oligomeric structure. The recruitment of this cargo into autophagosomes depends on Atg11 and the autophagy receptor Atg19 (Atg11 and Atg19 are not required for starvation-induced autophagy). The distribution of Atg19 on the surface of the cargo and its interaction with Atg8 through an LIR motif allow the hermetic formation of a double-membrane vesicle around the targeted structure. Atg32 and Atg36 are yeast autophagy receptors for mitochondria and peroxisomes, respectively; they are found on the surface of these organelles and seem to operate in the same way as Atg19 (refs 16-18).

An emerging theme in high eukaryotes is that structures targeted for destruction by autophagy are often ubiquitinated, and a series of autophagy receptors with a ubiquitin-binding domain and an LIR motif (such as p62/SQSTM1, NBR1, NDP52 or optineurin) promote their engulfment into autophagosomes ${ }^{13,19}$. With ubiquitination being a key regulatory element during selective types of autophagy, E3 ligases and/or their eventual adaptors and regulators must have central roles in these processes. However, only a handful of these molecules have been identified so far. Those include the Pink1 kinase, the Parkin ligase and the mitochondrial outer membrane protein FUNDC (all of which were linked to mitophagy ${ }^{20-22}$, and the SMURF1 ligase and the STING adaptor, which participate in the clearance of pathogens ${ }^{23,24}$.

\section{Post-translational modifications in the regulation of autophagy}

As for all degradative pathways, regulation is key to specifically 'switch on' autopahgy for the limited time that it is required. Several signalling molecules and cascades modulate autophagy in response to numerous cellular and environmental cues ${ }^{25,26}$. The best-characterized regulator of autophagy is mTOR complex 1 (mTORC1). This kinase negatively regulates autophagy by inhibiting the activity of the Atg1 (ULK1) complex through direct phosphorylation. The activity of mTORC1 is stimulated by a variety of anabolic inputs, which include the energy and nutrient status of the cell as well as the presence of amino acids and growth factors. Conversely, mTORC1 is inhibited when amino acids are scarce, growth factor signalling is reduced and/or ATP concentrations fall, and this results in a de-repression of autophagy ${ }^{26}$. 


\begin{tabular}{cccc} 
Protein & Yeast & High eukaryotes & Function \\
\hline Atg1/ULKs & + & + & Protein kinase involved in the induction of autophagy and possibly in PAS/phagophore biogenesis \\
Atg2 & + & + & Interacts with Atg18/WIPI4; possibly involved in PAS/phagophore biogenesis \\
Atg3 & + & + & E2-like enzyme for the ubiquitin-like conjugation system that catalyses Atg8/LC3's \\
lipidation involved in phagophore expansion
\end{tabular}

Atg4 ++

Atg5 ++

Atg6/beclin $1++$

$\begin{array}{lll}\text { Atg7 } & + \\ \text { Atg8/LC3s } & + \\ \text { Atg9 } & + \\ \text { Atg10 } & +\end{array}$

Atg12 + +

Atg13 + +

Atg14 + +

Atg16 + +

Atg17/FIP200 + + +

Atg18/WIPIs $\quad+\quad+$

Atg23 +

Atg27 +

$\operatorname{Atg} 29+$
$\operatorname{Atg} 31+$

Atg101 - + + +

Ambral $\quad-\quad+$

DFCP1 $\quad+$

VMP1 - +

$\operatorname{Vps} 15 / \mathrm{p} 150++$

Vps34/ PtdIns3PKC3

\begin{abstract}
Cysteine protease processing and delipidating Atg8/LC3, thus involved in phagophore expansion
Covalently linked to Atg12, generating the Atg12-Atg5 conjugate involved in phagophore expansion

Component of various $\mathrm{PI}(3) \mathrm{K}$ complexes, one of which is involved in induction of autophagy and PAS/phagophore biogenesis
\end{abstract}

E1-like enzyme for the two ubiquitin-like conjugation systems, thus involved in phagophore expansion

$$
\begin{aligned}
& \text { Ubiquitin-like protein involved in phagophore expansion } \\
& \begin{array}{l}
\text { Transmembrane protein involved in the induction of autophagy and possibly } \\
\text { in PAS/phagophore biogenesis }
\end{array}
\end{aligned}
$$

E2-like enzyme for the ubiquitin-like conjugation system that mediates the formation of the Atg12-Atg5 conjugate involved in phagophore expansion

Ubiquitin-like protein involved in phagophore expansion

Binding partner and regulator of Atg1/ULKs, thus involved in the induction of autophagy and possibly PAS/phagophore biogenesis

Component of the $\mathrm{PI}(3) \mathrm{K}$ complex I involved in induction of autophagy and possibly PAS/phagophore biogenesis

Associates with Atg12-Atg5 to form a large complex, which acts as an E3 ligase to direct LC3 lipidation on autophagosomal membranes, and thus involved in phagophore expansion

Binding partner and regulator of Atg1/ULKs, thus involved in the induction of autophagy and possibly PAS/phagophore biogenesis

PtsIns3P-binding proteins possibly involved in PAS/phagophore biogenesis

Binding partner and regulator of Atg9, thus involved in the induction of autophagy and possibly in PAS/phagophore biogenesis

Binding partner and regulator of Atg9, thus involved in the induction of autophagy and possibly in PAS/phagophore biogenesis

Binding partner and regulator of Atg1, thus involved in the induction of autophagy and possibly in PAS/phagophore biogenesis

Binding partner and regulator of Atg1, thus involved in the induction of autophagy and possibly in PAS/phagophore biogenesis

Binding partner and regulator of ULKs, thus involved in the induction of autophagy and possibly in PAS/phagophore biogenesis

Regulator of the PI(3)K and Atg1/ULK complexes, and thus involved in the induction of autophagy Ptdlns3P-binding proteins concentrating at the omegasome, possibly involved in the induction of autophagy

Transmembrane protein regulating autophagy induction

Kinase regulating Vps34/hVps34 activity; component of various $\mathrm{PI}(3) \mathrm{K}$ complexes, one of which is involved in the induction of autophagy and PAS/phagophore biogenesis

Component of various $\mathrm{PI}(3) \mathrm{K}$ complexes, one of which is involved in the induction of autophagy and PAS/phagophore biogenesis
The mTOR-dependent phosphorylation of the Atg1 (ULK1) complex and the ubiquitination-like reactions are central during autophagosome biogenesis through the generation of the Atg12-Atg5 and the Atg8phosphatidylethanolamine (PE) conjugates. Although these were initially considered to be the only post-translational modifications in autophagy, proteins linked to the Atg machinery are now known to be substrates for a wide range of post-translational modifications such as phosphorylation, ubiquitination and acetylation ${ }^{27}$.

Yeast protein kinase A (PKA) phosphorylates Atg13 in the presence of nutrients to prevent its association with the PAS (ref. 28). In mammalian cells, ULK1 is directly phosphorylated by the AMP-activated protein kinase (AMPK) in response to energy restriction ${ }^{29,30}$. Thus,
AMPK triggers autophagy by both positively regulating the Atg1/ (ULK1) complex and inhibiting mTOR (ref. 25).

The phosphatidylinositol-3-OH kinase $(\mathrm{PI}(3) \mathrm{K})$ complex I is also a major point of regulation for the kinases that modulate autophagy induction. Beclin 1 is one of the subunits of the PI(3)K complex I and its incorporation into this complex, which is essential to stimulate PtdIns3P synthesis, is usually kept in check by its association with other proteins, such as Bcl-2, 14-3-3 or the intermediate filament protein vimentin 1 (VMP1). The phosphorylation of beclin 1 by the deathassociated protein kinase (DAPK) or phosphorylation of Bcl-2 by the c-Jun N-terminal kinase (JNK) triggers the dissociation of the beclin-1-Bcl-2 complex in response to various stimuli, allowing beclin 1 
a

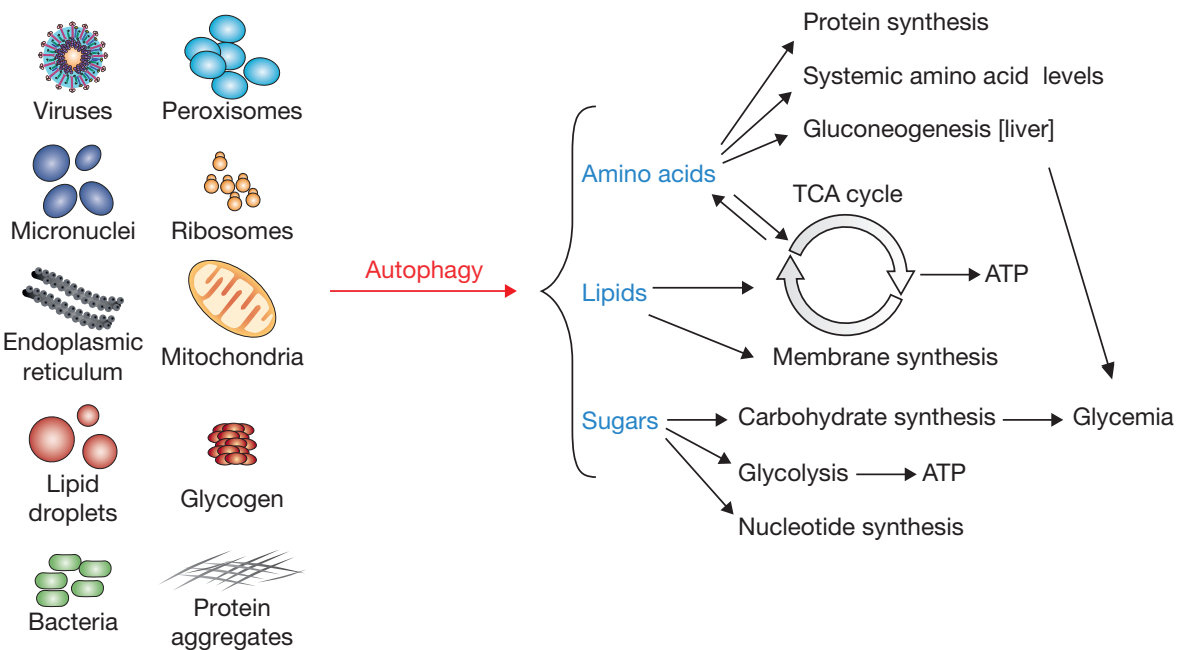

b

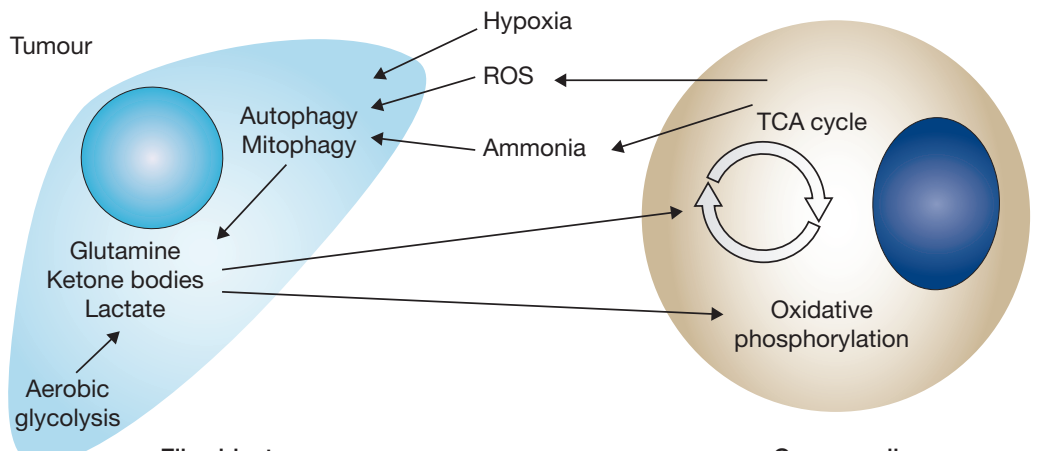

Fibroblast

Cancer cell

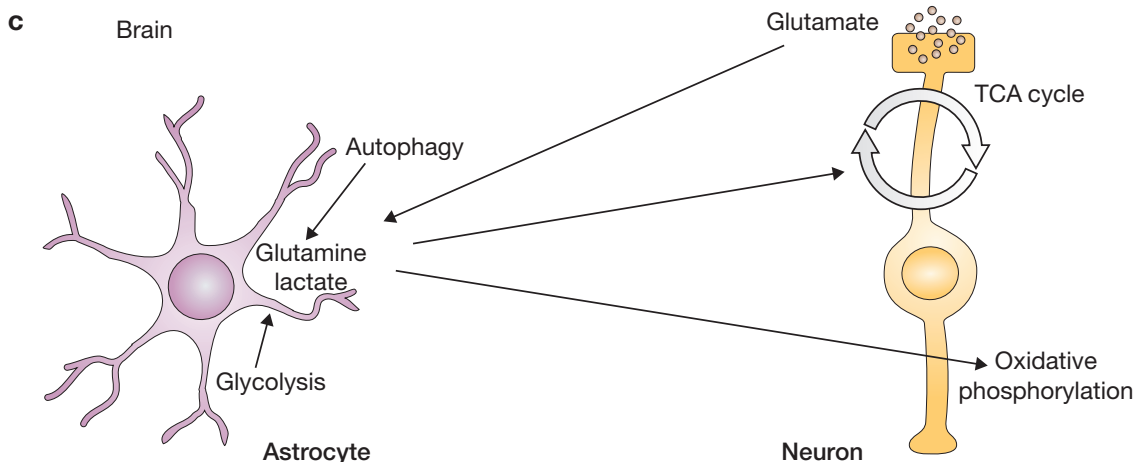

Figure 2 Relationship between autophagy and the main metabolic pathways. (a) The catabolic products of the intracellular structures that are targeted by autophagosomes, such as amino acids, lipids and sugars, are used for anabolic reactions to generate new proteins, glycans, oligonucleotides and membranes to sustain cell functions. Amino acids can also be used to maintain their systemic levels and for de novo synthesis of glycogen (gluconeogenesis) in the liver. Lipids and amino acids can enter the tricarboxylic acid (TCA) cycle and oxidative phosphorylation to generate energy in the form of ATP. Sugars can also be metabolized to generate ATP through glycolysis and to maintain systemic glucose levels. (b,c) Metabolic compartmentalization between different cell types. (b) Inside tumours, hypoxia and oxidative stress trigger autophagy and mitophagy in the stromal fibroblasts. This induces a metabolic switch towards aerobic glycolysis (known as the Warburg effect), leading to the production of lactate and other metabolites that are liberated into the intracellular space and reabsorbed by tumour cells. A more oxidative metabolism in these cells generates oxidative stress and ammonia (from glutaminolysis), which signals back to fibroblasts to further stimulate autophagy. (c) In brain tissue, astrocytes produce lactate from glucose through glycolysis and glutamine through autophagy. These metabolites are taken up by neurons and oxidized to generate ATP. Moreover, the neurotransmitter glutamate, released by neurons, can be retransformed into glutamine by astrocytes.

to associate with the PI(3)K complex I (refs 31,32). More recently, it has been shown that beclin 1 phopshorylation by Akt and protein kinase $B$ $(\mathrm{PKB})$ inhibits autophagy by favouring the interaction of beclin 1 with 14-3-3 and the vimentin intermediate filament protein 1 (ref. 33).
Furthermore, AMPK stimulates autophagy in response to glucose starvation by phosphorylating beclin 1 on a different residue to that of the inhibitory kinases, a modification that promotes its incorporation into the PI(3)K complex I (ref. 34). 
Downstream of the ULK and the PI(3)K complexes, phosphorylation can regulate the activity of LC3-II. When phosphorylated by PKA or protein kinase $\mathrm{C}$ ( $\mathrm{PKC})$, LC3 becomes inoperative in autophagosome forma$\operatorname{tion}^{28,35}$. How exactly phosphorylation inhibits LC3 function remains to be elucidated. The PKA site is highly conserved in the human, mouse and rat isoforms of LC3, but is not present in yeast Atg8 (ref. 28).

Phosphorylation of autophagy receptors also increases their affinity for binding to substrates and LC3 during selective types of autophagy. Phosphorylation of p62 by the casein kinase 2 favours its interaction with ubiquitinated proteins ${ }^{36}$, whereas phosphorylation of optineurin by the TANK-binding kinase 1 enhances its affinity for LC3 to promote the elimination of cytosolic Salmonella ${ }^{37}$.

Although ubiquitination plays an important role in the selection of the cargo targeted for destruction during selective types of autophagy, there are so far no indications that this post-translational modification occurs on Atg proteins. Recently, the formation of an Atg12-Atg3 conjugate though the action of Atg7 and the autocatalytic activity of Atg3 has been described in mammalian cells ${ }^{38}$. This conjugate plays a role in cell death pathways and in the control of mitochondrial expansion.

An initial study showed that some of the Atg proteins are acetylated ${ }^{39}$ and an analysis of the acetylome has revealed the importance of this modification in the regulation of autophagy. Atg5, Atg7, LC3 and Atg12 are acetylated by the p300 acetyltransferase when cells are maintained in nutrient-rich media and deacetylated by Sirt1 in response to starvation, an event necessary to induce autophagy ${ }^{40,41}$. More recently, the acetylation of Atg 3 by Esa1 in yeast and by the Esal orthologue TIP60 in mammals under autophagy-inducing conditions has been shown to promote the interaction between Atg3 and Atg8, which is required for Atg8 lipidation ${ }^{42}$. TIP60 also acetylates ULK1 in a glycogen synthase kinase-dependent manner in response to growth factor deprivation ${ }^{43}$. Thus, ULK1 is activated by either phosphorylation or acetylation in response to amino acid (or glucose) and growth factor deprivation, respectively ${ }^{44}$.

Autophagy and Atg proteins in membrane transport and secretion Until recently, the Cvt pathway has been the only transport pathway depending on Atg proteins that is not associated with degradation. The Atg machinery has now been shown to participate in the release of cargoes into the extracellular medium, shedding light on the mechanism of a few types of unconventional protein secretion ${ }^{45-47}$. This mechanism seems to be involved in the secretion of the pro-inflammatory cytokines IL-1 and IL-18 in mammalian cells ${ }^{47}$. This process depends on Atg5, the inflammasome, the peripheral Golgi protein GRASP55 and the small GTPase Rab8a. This autophagy-based unconventional secretion mechanism can probably be extended to modulators of the immune response such as HMGB1 (ref. 47). The morphology of the autophagy-related organelles involved in secretion and the molecular basis for their formation, however, remain to be identified. In yeast, the putative carriers for this type of secretion were suggested to come from a hitherto-unknown compartment for unconventional protein secretion (aptly named CUPS) ${ }^{48}$. Like the PAS, CUPS also contains PtdIns3P as well as Atg8 and Atg9. However, although these two Atg proteins are required for the generation of the PAS, they seem to be unnecessary for CUPS formation. In mammalian systems, this mode of secretion could explain the non-lytic release of viruses that subverts the autophagy machinery for egression ${ }^{49}$ or the expulsion of cellular material by autophagosomes at the late stages of reticulocyte maturation into erythrocytes ${ }^{50}$.

In oncogene-induced senescent cells, a specialized compartment known as the mTOR-autophagy special coupling compartment (TASCC) is juxtaposed next to the Golgi apparatus and stimulates the extracellular release of a specific subset of proteins through the conventional secretory pathway ${ }^{51}$. Lysosomes and autophagosomes (which supply lysosomes with proteins) both accumulate adjacent to the TASCC. Importantly, mTOR located at the lysosomal surface is activated by amino acid efflux from lysosomes and positively regulates protein synthesis and cell growth $^{52}$. Interactions between TASCC, lysosomes and autophagosomes could be key in coordinating cell metabolism by coupling autophagic degradation with both the synthesis and secretion of proteins. TASCClike structures have also been observed in non-senescent cells, suggesting that this mechanism for protein secretion may be widely used ${ }^{51}$.

Autophagy and Atg proteins, including the Atg8 conjugate LC3-II, also modulate secretion in several specialized tissues including the middle ear, osteoclasts, mast cells, Paneth cells and pancreatic $\beta$-cells ${ }^{53}$. Independently of autophagosome formation, LC3-II mediates the fusion of vesicular carriers containing the protease cathepsin $\mathrm{K}$ with the ruffled border of osteoclasts, which is an important step in bone resorption ${ }^{54}$. This situation mirrors the recruitment of LC3-II on to phagosomes to enhance their fusion with lysosomes in phagocytic cells ${ }^{55}$. The role of LC3-II and other members of the Atg8 protein family in these processes probably relies on their capacities in mediating tethering or fusion of vesicular structures ${ }^{56,57}$ and/or binding the cytoskeleton ${ }^{58}$.

Autophagy as a regulator of tissue microenvironment metabolism Starvation is the classical autophagy stimulus that induces the degradation of intracellular components to generate metabolites essential to maintain cell viability (Fig. 2a), which are used to fuel mitochondrial respiration and ATP production ${ }^{59,60}$. In yeast, amino acids generated by autophagy can be used to sustain new protein synthesis and to maintain mitochondrial functions under nutrient deprivation ${ }^{61}$. Amino acids generated in the liver by autophagy are used for gluconeogenesis to maintain systemic glycemia under starvation (Fig. 2a) ${ }^{62}$. Moreover, in addition to the activity of hepatic triglyceride lipases, the selective degradation of lipid droplets in the liver by autophagy also produces free fatty acids from triglycerides ${ }^{63}$.

The role of autophagy in cancer metabolism has been the subject of intense investigation. Autophagy protects cancer cells from metabolic stress (such as decreased nutrient availability and hypoxic conditions) by reducing oxidative stress and maintaining genomic stability ${ }^{64}$. It is now clear that cancer cells reprogram their metabolism to support their rapid proliferation and growth. To increase their nutrient uptake, cancer cells also perform aerobic glycolysis to oxidize glucose into lactate to produce ATP and intermediary metabolites used for anabolic reactions that sustain cell growth ${ }^{65}$. This metabolic switch includes the expression of specific isoforms of glycolytic enzymes, as well as expression of enzymes metabolizing amino acids and lipids, with distinct enzymatic activities and substrate preferences ${ }^{66}$. In this context, the activation of the hypoxia-responsive factor HIF-1 regulates the expression of many glycolytic enzymes and also induces mitophagy ${ }^{67}$. A recent metabolomic study has demonstrated that cells can maintain minimal levels of mitochondrial respiration to produce ATP under hypoxia (1\% oxygen), and these cells also display increased levels of autophagy activity and 
Table 2 Detailed list of the ATG genes that have been implicated in non-autophagic pathways

\begin{tabular}{|c|c|c|}
\hline Gene & Autophagy-independent pathways & References \\
\hline ULK1 & Brucella vacuole biogenesis & 80 \\
\hline ATG3 & Mitochondrial homeostasis (conjugated to Atg12) & 38 \\
\hline ATG4B & Osteoclast bone resorption & 54 \\
\hline ATG5 & $\begin{array}{l}\text { Osteoclast bone resorption; phagocytosis; IFN- } \alpha / \text { IFN- } \beta / \text { IFN- } \gamma \text { antiviral response (conjugated to Atg12); } \\
\text { pro-apoptotic role }\end{array}$ & $54,55,77,78,84,94$ \\
\hline Beclin 1 & Phagocytosis; Brucella vacuole biogenesis & 80,95 \\
\hline ATG12 & $\begin{array}{c}\text { Mitochondrial apoptosis; IFN- } / \text { /IFN- } \beta / / F N-\gamma \text { antiviral response (conjugated to Atg5); } \\
\text { mitochondrial homeostasis (conjugated to Atg3) }\end{array}$ & $38,77,78,83$ \\
\hline ATG14 & Brucella vacuole biogenesis & 80 \\
\hline ATG16 & IFN- $\gamma$ antiviral response & 78 \\
\hline p150 & Brucella vacuole biogenesis & 80 \\
\hline
\end{tabular}

intermediates resulting from protein and lipid catabolism ${ }^{68}$. Autophagy inhibition under these conditions reduces intracellular ATP levels and induces cell death, indicating that the degradation products resulting from autophagy are able to fuel the tricarboxylic acid cycle for ATP synthesis to maintain cell viability even under low oxygen conditions ${ }^{68}$. This cyto-protective role of autophagy under hypoxic conditions may be modulated through microRNA-dependent regulation of ATG7 expression in hepatic tumour cell lines and in vivo xenographs ${ }^{69}$. It is still unknown whether the autophagy-dependent degradation of mitochondria is an additional mechanism responsible for metabolic reprogramming, although observations in non-tumorigenic cells would support this notion ${ }^{70}$. Indeed, overexpression of the RCAN1-1L protein induces mitophagy and a shift from oxidative to glycolytic metabolism in neuronal cells ${ }^{70}$.

Metabolic coupling, a phenomenon in which two different cell types differentially coordinate their metabolism, has been associated with autophagy and has been observed in several tissues including tumours and brain tissue (Fig. 2b,c) ${ }^{71}$. Tumours are composed of several cell types, and it has been postulated that metabolic coupling is essential for tumour development. In particular, fibroblasts perform aerobic glycolysis inside tumours and display an increased expression of glycolytic enzymes, elevated HIF-1 activity, autophagy and mitophagy ${ }^{71}$. Metabolites resulting from elevated autophagy and glycolysis in these fibroblasts, such as lactate, ketone bodies and amino acids, are released into the tumour microenvironment and sequestered by cancer cells to fuel the oxidative phosphorylation necessary to sustain tumour growth ${ }^{71}$. The autophagy stimulation in fibroblasts also results in increased senescence, which boosts both the production of ketone bodies and mitochondrial metabolism in adjacent cancer cells to promote metastasis ${ }^{72}$. In contrast to these positive effects of autophagy on tumour growth, autophagy upregulation in the cancer cells themselves actually inhibit tumour growth ${ }^{72}$. Other metabolites, such as the ammonia generated from glutaminolysis in cancer cells, stimulate autophagy in neighbouring cells ${ }^{73}$.

Similar features of metabolism coupling have been observed in the brain, where astrocytes and neurons exchange metabolites to support their cellular functions (Fig. 2c). Impairment of lysosomal functions and autophagy affects specific cell types such as astrocytes, preventing them from supporting and protecting neighbouring neurons, ultimately resulting in cortical neurodegeneration in vivo $^{74}$.
Together, these data indicate that autophagy in specific cell types could be key in regulating the survival and growth of the surrounding tissue (Fig. 2c).

\section{The role of $A T G$ genes in other cellular processes}

Analysis of the Atg protein interactome suggested that they can function in cellular pathways independently of their role in autophagy ${ }^{75}$. This emerging topic has been recently reviewed $^{76}$, and some of the non-autophagic functions of ATG genes are shown in Table 2. Some modules involved in autophagosome formation, such as the two conjugation systems (ATG5-ATG12 and LC3-PE) and the PI(3)K complex I, are recruited to the phagosomal membrane to promote the fusion between phagosomes and lysosomes during phagocytosis triggered by the engagement of Toll-like receptors ${ }^{55}$. These conjugation systems are also required for the generation of the osteoclast ruffled border, a key structure for bone resorption ${ }^{54}$.

The ATG5-ATG12/ATG16L1 complex, independently of the LC3 conjugation system, can modulate the innate viral immune response. ATG5-ATG12 suppresses the type I interferon (IFN) production by direct association with the retinoic-acid-inducible gene I (RIG-I) and IFN- $\beta$ promoter stimulator (IPS-1) $)^{77}$. This complex is also required for type II IFNmediated host defense against norovirus by inhibiting the formation of its membranous replication complex ${ }^{78}$. Similarly, ATG5 is required for the clearance of the parasite Toxoplasma gondii in macrophages ${ }^{79}$.

Complexes acting upstream of the conjugation systems, such as the ULK1 complex and PI(3)K complex I, are necessary for the intracellular cycle of the bacterium Brucella abortus. They are used to subvert clearance by participating in the formation of a vacuole containing the bacteria that promote infection ${ }^{80}$.

In addition to the conventional ATG12-ATG5 cassette, an ATG12ATG3 conjugate has been shown to regulate mitochondria homeostasis and cell death without affecting the formation of autophagosomes in response to starvation ${ }^{38}$. Both LC3 and ATG12 can also function independently of their conventional conjugation to PE and ATG5, respectively. LC3-I is involved in the formation of carriers derived from the endoplasmic reticulum, called EDEMosomes ${ }^{81}$, a pathway hijacked by coronaviruses to form structures needed for the transcription and replication of the viral genome ${ }^{82}$. ATG12 is a positive mediator of mitochondrial apoptosis by inactivating members of the pro-survival 
Bcl-2 protein family. The activity of ATG12 is independent of ATG5 or ATG3, and requires a BH3-like motif in ATG12 (ref. 83). ATG proteins also act in an autophagy-independent manner following proteolytic processing. For example, ATG5 cleavage by calpains generates a pro-apoptotic fragment that interferes with the anti-apoptotic activity of $\mathrm{Bcl}-\mathrm{xL}$ (ref. 84). Moreover, beclin 1 processing by caspase 3 generates two fragments that do not have the capacity to induce autophagy ${ }^{85,86}$. The C-terminal fragment resulting from this processing localizes to mitochondria and sensitizes cells to apoptosis ${ }^{86}$. Thus, similarly to proteins involved in apoptosis that also function beyond apoptosis ${ }^{87}$, ATG components, as well as other proteins involved in autophagy such as AMBRA1, VPS34 and p62 (for which autophagy-independent roles are not discussed here), are engaged in non-autophagic functions. This notion has to be taken into account when we experimentally explore the role of autophagy in vivo on the basis of the ablation of a single ATG gene.

\section{Conclusions and perspectives}

Despite the progresses made in our understanding of autophagy, numerous key aspects of this catabolic pathway remain enigmatic. For example, we still know very little about the regulation of basal autophagy, which operates under normal growing conditions. The modulation of this process engages actin filaments and the histone deacetylase HDAC6, which are both dispensable for autophagosome maturation under starvation conditions ${ }^{88}$.

The coordination of Atg protein recruitment to the PAS from different membrane origins, as well as their hierarchical assembly at this specialized site, are aspects of the autophagosome biogenesis that should be carefully considered in the future. Although a hierarchical recruitment of the Atg proteins has been proposed for yeast on a genetic basis ${ }^{89}$, their temporal association and the functional consequences of the hierarchy remain to be investigated. Examination of the selective sequestration of mitochondria or Salmonella by autophagosomes has indicated a different hierarchy than the one postulated, according to which groups or clusters of Atg proteins could independently associate to form the PAS (refs 90,91). Similarly, further analyses of the different forms of noncanonical autophagy described to date, which only require a subset of Atg proteins, or of the autophagy-independent functions of Atg proteins, can yield a better understanding of the functional importance of these factors during the generation of autophagosomes ${ }^{92}$.

It remains to be seen why metazoans possess several isoforms of the same Atg protein, which apparently have identical functions. For example, the human genome contains 6 homologues of the single yeast Atg8 protein, yet the role of these counterparts remains to be elucidated. Some members of this protein family, that is, GABARAPL1 and GABARAPL2, were shown to participate in autophagosome closure ${ }^{57}$, whereas LC3C also acts as a specific receptor during selective antibacterial autophagy ${ }^{93}$.

The importance of autophagy in many aspects of physiology is now recognised. Not surprisingly, defects in this process are intimately associated with numerous human diseases. Better knowledge of the molecular bases of autophagy and of its control by physiological regulators, from cytokines and hormones to dietary restriction or physical exercise, may provide simple and non-invasive ways to modulate this pathway for preventive or therapeutic interventions in the future ${ }^{25}$.

\section{ACKNOWLEDGEMENTS}

P.B. is supported by the SAF-2009-08086 and SAF-2012-36079 grants. F.R. is supported by the ECHO (700.59.003), ALW Open Program (821.02.017 and
822.02.014), DFG-NWO cooperation (DN82-303) and ZonMW VICI (016.130.606) grants. P.C. is supported by INSERM and grants from ANR and INCa.

1. Yang, Z. \& Klionsky, D. J. Eaten alive: a history of macroautophagy. Nat. Cell Biol. 12, 814-822 (2010).

2. Mizushima, N., Yoshimori, T. \& Ohsumi, Y. The role of Atg proteins in autophagosome formation. Annu. Rev. Cell Dev. Biol. 27, 107-132 (2011).

3. Rubinsztein, D. C., Shpilka, T. \& Elazar, Z. Mechanisms of autophagosome biogenesis. Curr. Biol. 22, R29-34 (2012).

4. Axe, E. L. et al. Autophagosome formation from membrane compartments enriched in phosphatidylinositol 3-phosphate and dynamically connected to the endoplasmic reticulum. J. Cell Biol. 182, 685-701 (2008).

5. Hayashi-Nishino, M. et al. A subdomain of the endoplasmic reticulum forms a cradle for autophagosome formation. Nat. Cell Biol. 11, 1433-1437 (2009).

6. Yla-Anttila, P., Vihinen, H., Jokitalo, E. \& Eskelinen, E. L. 3D tomography reveals connections between the phagophore and endoplasmic reticulum. Autophagy 5, 1180-1185 (2009).

7. Hailey, D. W. et al. Mitochondria supply membranes for autophagosome biogenesis during starvation. Cell 141, 656-667 (2010).

8. Deretic, V. \& Levine, B. Autophagy, immunity, and microbial adaptations. Cell Host Microbe 5, 527-549 (2009).

9. Mizushima, N., Levine, B., Cuervo, A. M. \& Klionsky, D. J. Autophagy fights disease through cellular self-digestion. Nature 451, 1069-1075 (2008).

10. Levine, B. \& Kroemer, G. Autophagy in the pathogenesis of disease. Cel/ 132, 27-42 (2008).

11. Reggiori, F., Komatsu, M., Finley, K. \& Simonsen, A. Autophagy: more than a nonselective pathway. Int. J. Cell Biol. 2012, 219625 (2012).

12. Shi, C. S. et al. Activation of autophagy by inflammatory signals limits IL-1 $\beta$ production by targeting ubiquitinated inflammasomes for destruction. Nat. Immunol. 13, 255-263 (2012).

13. Johansen, T. \& Lamark, T. Selective autophagy mediated by autophagic adapter proteins. Autophagy 7, 279-296 (2011).

14. Noda, N. N., Ohsumi, Y. \& Inagaki, F. Atg8-family interacting motif crucial for selective autophagy. FEBS Lett. 584, 1379-1385 (2010)

15. Lynch-Day, M. A. et al. Trs85 directs a Ypt1 GEF, TRAPPIII, to the phagophore to promote autophagy. Proc. Natl Acad. Sci. USA 107, 7811-7816 (2010).

16. Motley, A. M., Nuttall, J. M. \& Hettema, E. H. Pex3-anchored Atg36 tags peroxisomes for degradation in Saccharomyces cerevisiae. EMBO J. 31, 2852-2868 (2012).

17. Kanki, T., Wang, K., Cao, Y., Baba, M. \& Klionsky, D. J. Atg32 is a mitochondrial protein that confers selectivity during mitophagy. Dev. Cell 17, 98-109 (2009).

18. Okamoto, K., Kondo-Okamoto, N. \& Ohsumi, Y. Mitochondria-anchored receptor Atg32 mediates degradation of mitochondria via selective autophagy. Dev. Cell 17, 87-97 (2009).

19. Shaid, S., Brandts, C. H., Serve, H. \& Dikic, I. Ubiquitination and selective autophagy. Cell Death Differ. 20, 21-30 (2013).

20. Vives-Bauza, C. et al. PINK1-dependent recruitment of Parkin to mitochondria in mitophagy. Proc. Natl Acad. Sci. USA 107, 378-383 (2010).

21. Narendra, D., Tanaka, A., Suen, D. F. \& Youle, R. J. Parkin is recruited selectively to impaired mitochondria and promotes their autophagy. J. Cell Biol. 183, 795-803 (2008).

22. Liu, L. et al. Mitochondrial outer-membrane protein FUNDC1 mediates hypoxia-induced mitophagy in mammalian cells. Nat. Cell Biol. 14, 177-185 (2012).

23. Watson, R. O., Manzanillo, P. S. \& Cox, J. S. Extracellular M. tuberculosis DNA targets bacteria for autophagy by activating the host DNA-sensing pathway. Cel/ 150, 803-815 (2012).

24. Orvedahl, A. et al. Image-based genome-wide siRNA screen identifies selective autophagy factors. Nature 480, 113-117 (2011).

25. Rubinsztein, D. C., Codogno, P. \& Levine, B. Autophagy modulation as a potential therapeutic target for diverse diseases. Nat. Rev. Drug Discov. 11, 709-730 (2012).

26. Meijer, A. J. \& Codogno, P. Autophagy: regulation and role in disease. Crit. Rev. Clin. Lab. Sci. 46, 210-240 (2009).

27. McEwan, D. G. \& Dikic, I. The Three Musketeers of Autophagy: phosphorylation, ubiquitylation and acetylation. Trends Cell Biol. 21, 195-201 (2011).

28. Stephan, J. S., Yeh, Y. Y., Ramachandran, V., Deminoff, S. J. \& Herman, P. K. The Tor and PKA signaling pathways independently target the Atg1/Atg13 protein kinase complex to control autophagy. Proc. Natl Acad. Sci. USA 106, 17049-17054 (2009).

29. Egan, D. F. et al. Phosphorylation of ULK1 (hATG1) by AMP-activated protein kinase connects energy sensing to mitophagy. Science 331, 456-461 (2011).

30. Kim, J., Kundu, M., Viollet, B. \& Guan, K. L. AMPK and mTOR regulate autophagy through direct phosphorylation of Ulk1. Nat. Cell Biol. 13, 132-141 (2011).

31. Wei, Y., Pattingre, S., Sinha, S., Bassik, M. \& Levine, B. JNK1-mediated phosphorylation of $\mathrm{Bcl}-2$ regulates starvation-induced autophagy. Mol. Cell 30, 678-688 (2008).

32. Zalckvar, E. et al. DAP-kinase-mediated phosphorylation on the $\mathrm{BH} 3$ domain of beclin 1 promotes dissociation of beclin 1 from $\mathrm{Bcl}-\mathrm{X}(\mathrm{L})$ and induction of autophagy. EMBO Rep. 30, 30 (2009).

33. Wang, R. C. et al. Akt-mediated regulation of autophagy and tumorigenesis through Beclin 1 phosphorylation. Science 338, 956-959 (2012).

34. Kim, J. et al. Differential regulation of distinct Vps34 complexes by AMPK in nutrient stress and autophagy. Cell 152, 290-303 (2013).

35. Cherra, S. J., 3rd. et al. Regulation of the autophagy protein LC3 by phosphorylation. J. Cell Biol. 190, 533-539 (2010).

36. Matsumoto, G., Wada, K., Okuno, M., Kurosawa, M. \& Nukina, N. Serine 403 phosphorylation of p62/SQSTM1 regulates selective autophagic clearance of ubiquitinated proteins. Mol. Cell 44, 279-289 (2011). 
37. Wild, P. et al. Phosphorylation of the autophagy receptor optineurin restricts Salmonella growth. Science 333, 228-233 (2011).

38. Radoshevich, L. et al. ATG12 conjugation to ATG3 regulates mitochondrial homeostasis and cell death. Cell 142, 590-600 (2010).

39. Morselli, E. et al. Spermidine and resveratrol induce autophagy by distinct pathways converging on the acetylproteome. J. Cell Biol. 192, 615-629 (2011).

40. Lee, I. H. et al. A role for the NAD-dependent deacetylase Sirt1 in the regulation of autophagy. Proc. Natl Acad. Sci. USA 105, 3374-3379 (2008).

41. Lee, I. H. \& Finkel, T. Regulation of autophagy by the p300 acetyltransferase. J. Biol. Chem. 284, 6322-6328 (2009).

42. Yi, C. et al. Function and molecular mechanism of acetylation in autophagy regulation. Science 336, 474-477 (2012).

43. Lin, S. Y. et al. GSK3-TIP60-ULK1 signaling pathway links growth factor deprivation to autophagy. Science 336, 477-481 (2012).

44. Hamai, A. \& Codogno, P. New targets for acetylation in autophagy. Sci. Signal. 5, pe29 (2012).

45. Duran, J. M., Anjard, C., Stefan, C., Loomis, W. F. \& Malhotra, V. Unconventional secretion of Acb1 is mediated by autophagosomes. J. Cell Biol. 188, 527-536 (2010).

46. Manjithaya, R., Anjard, C., Loomis, W. F. \& Subramani, S. Unconventional secretion of Pichia pastoris Acb1 is dependent on GRASP protein, peroxisomal functions, and autophagosome formation. J. Cell Biol. 188, 537-546 (2010).

47. Dupont, N. et al. Autophagy-based unconventional secretory pathway for extracellular delivery of IL-1 $\beta$. Embo J 30, 4701-4711 (2011)

48. Bruns, C., McCaffery, J. M., Curwin, A. J., Duran, J. M. \& Malhotra, V. Biogenesis of a novel compartment for autophagosome-mediated unconventional protein secretion. J. Cell Biol. 195, 979-992 (2011).

49. Jackson, W. T. et al. Subversion of cellular autophagosomal machinery by RNA viruses. PLoS Biol. 3, e156 (2005).

50. Griffiths, R. E. et al. Maturing reticulocytes internalize plasma membrane in glycophorin A-containing vesicles that fuse with autophagosomes before exocytosis. Blood 119, 6296-6306 (2012).

51. Narita, M. et al. Spatial coupling of mTOR and autophagy augments secretory phenotypes. Science 332, 966-970 (2011).

52. Zoncu, R. et al. mTORC1 senses lysosomal amino acids through an inside-out mechanism that requires the vacuolar H(+)-ATPase. Science 334, 678-683 (2011).

53. Deretic, V., Jiang, S. \& Dupont, N. Autophagy intersections with conventional and unconventional secretion in tissue development, remodeling and inflammation. Trends Cell Biol. 22, 397-406 (2012).

54. DeSelm, C. J. et al. Autophagy proteins regulate the secretory component of osteoclastic bone resorption. Dev. Cell 21, 966-974 (2011).

55. Sanjuan, M. A. et al. Toll-like receptor signalling in macrophages links the autophagy pathway to phagocytosis. Nature 450, 1253-1257 (2007).

56. Nakatogawa, H., Ichimura, Y. \& Ohsumi, Y. Atg8, a ubiquitin-like protein required for autophagosome formation, mediates membrane tethering and hemifusion. Cell 130, 165-178 (2007).

57. Weidberg, H. et al. LC3 and GATE-16 N termini mediate membrane fusion processes required for autophagosome biogenesis. Dev. Cel/ 20, 444-454 (2011).

58. Monastyrska, I., Rieter, E., Klionsky, D. J. \& Reggiori, F. Multiple roles of the cytoskeleton in autophagy. Biol. Rev. Camb. Philos. Soc. 84, 431-448 (2009).

59. Mellén, M. A., de la Rosa, E. J. \& Boya, P. The autophagic machinery is necessary for removal of cell corpses from the developing retinal neuroepithelium. Cell Death Differ. $15,1279-1290$ (2008)

60. Qu, X. et al. Autophagy gene-dependent clearance of apoptotic cells during embryonic development. Cell 128, 931-946 (2007).

61. Suzuki, S. W., Onodera, J. \& Ohsumi, Y. Starvation induced cell death in autophagydefective yeast mutants is caused by mitochondria dysfunction. PloS One 6, e17412 (2011).

62. Ezaki, J. et al. Liver autophagy contributes to the maintenance of blood glucose and amino acid levels. Autophagy 7, 727-736 (2011).

63. Singh, R. et al. Autophagy regulates lipid metabolism. Nature 458, 1131-1135 (2009).

64. White, E. Deconvoluting the context-dependent role for autophagy in cancer. Nat. Rev. Cancer 12, 401-410 (2012)

65. Lunt, S. Y. \& Vander Heiden, M. G. Aerobic glycolysis: meeting the metabolic requirements of cell proliferation. Annu. Rev. Cell Dev. Biol. 27, 441-464 (2011).

66. Porporato, P. E., Dhup, S., Dadhich, R. K., Copetti, T. \& Sonveaux, P. Anticancer targets in the glycolytic metabolism of tumors: a comprehensive review. Front Pharmacol. 2 , 49 (2011).

67. Semenza, G. L. HIF-1: upstream and downstream of cancer metabolism. Curr. Opin. Genet. Dev. 20, 51-56 (2010).
68. Frezza, C. et al. Metabolic profiling of hypoxic cells revealed a catabolic signature required for cell survival. PloS One 6, e24411 (2011).

69. Chang, Y. et al. miR-375 inhibits autophagy and reduces viability of hepatocellular carcinoma cells under hypoxic conditions. Gastroenterology 143, 177-187 (2012).

70. Ermak, G. et al. Chronic expression of RCAN1-1L protein induces mitochondrial autophagy and metabolic shift from oxidative phosphorylation to glycolysis in neuronal cells. J. Biol. Chem. 287, 14088-14098 (2012).

71. Pavlides, S. et al. Warburg meets autophagy: cancer-associated fibroblasts accelerate tumor growth and metastasis via oxidative stress, mitophagy, and aerobic glycolysis. Antioxid. Redox Signal. 16, 1264-1284 (2012).

72. Capparelli, C. et al. Autophagy and senescence in cancer-associated fibroblasts metabolically supports tumor growth and metastasis via glycolysis and ketone production. Cell Cycle 11, 2285-2302 (2012).

73. Eng, C. H. \& Abraham, R. T. Glutaminolysis yields a metabolic by-product that stimulates autophagy. Autophagy 6, 968-970 (2010).

74. Di Malta, C., Fryer, J. D., Settembre, C. \& Ballabio, A. Astrocyte dysfunction triggers neurodegeneration in a lysosomal storage disorder. Proc. Natl Acad. Sci. USA 109, E2334-2342 (2012).

75. Behrends, C., Sowa, M. E., Gygi, S. P. \& Harper, J. W. Network organization of the human autophagy system. Nature 466, 68-76 (2010).

76. Subramani, S. \& Malhotra, V. Non-autophagic roles of autophagy-related proteins. EMBO Rep. 14, 143-151 (2013).

77. Jounai, N. et al. The Atg5 Atg12 conjugate associates with innate antiviral immune responses. Proc. Natl Acad. Sci. USA 104, 14050-14055 (2007).

78. Hwang, S. et al. Nondegradative role of Atg5-Atg12/Atg16L1 autophagy protein complex in antiviral activity of interferon gamma. Cell Host Microbe 11, 397-409 (2012).

79. Zhao, Z. et al. Autophagosome-independent essential function for the autophagy protein Atg5 in cellular immunity to intracellular pathogens. Cell Host Microbe 4, 458-469 (2008).

80. Starr, T. et al. Selective subversion of autophagy complexes facilitates completion of the Brucella intracellular cycle. Cell Host Microbe 11, 33-45 (2012).

81. Cali', T., Galli, C., Olivari, S. \& Molinari, M. Segregation and rapid turnover of EDEM 1 by an autophagy-like mechanism modulates standard ERAD and folding activities. Biochem. Biophys. Res. Commun. 371, 405-410 (2008).

82. Reggiori, F. et al. Coronaviruses hijack the LC3-I-positive EDEMosomes, ER-derived vesicles exporting short-lived ERAD regulators, for replication. Cell Host Microbe 7, 500-508 (2010).

83. Rubinstein, A. D., Eisenstein, M., Ber, Y., Bialik, S. \& Kimchi, A. The autophagy protein Atg12 associates with anti-apoptotic Bcl-2 family members to promote mitochondrial apoptosis. Mol. Cell 44, 698-709 (2011).

84. Yousefi, S. et al. Calpain-mediated cleavage of Atg 5 switches autophagy to apoptosis. Nat. Cell Biol. 8, 1124-1132 (2006).

85. Luo, S. \& Rubinsztein, D. C. Apoptosis blocks Beclin 1-dependent autophagosome synthesis: an effect rescued by Bcl-xL. Cell Death Differ. 17, 268-277 (2010).

86. Wirawan, E. et al. Caspase-mediated cleavage of Beclin-1 inactivates Beclin-1-induced autophagy and enhances apoptosis by promoting the release of proapoptotic factors from mitochondria. Cell Death Dis. 1, e18 (2010).

87. Galluzzi, L., Kepp, O., Trojel-Hansen, C. \& Kroemer, G. Non-apoptotic functions of apoptosis-regulatory proteins. EMBO Rep. 13, 322-330 (2012).

88. Lee, J. Y. et al. HDAC6 controls autophagosome maturation essential for ubiquitinselective quality-control autophagy. Embo J. 29, 969-980 (2010).

89. Suzuki, K., Kubota, Y., Sekito, T. \& Ohsumi, Y. Hierarchy of Atg proteins in preautophagosomal structure organization. Genes Cells 12, 209-218 (2007).

90. Itakura, E., Kishi-Itakura, C., Koyama-Honda, I. \& Mizushima, N. Structures containing Atg9A and the ULK1 complex independently target depolarized mitochondria at initial stages of Parkin-mediated mitophagy. J. Cell Sci. 125, 1488-1499 (2012).

91. Kageyama, S. et al. The LC3 recruitment mechanism is separate from Atg9L1dependent membrane formation in the autophagic response against Salmonella. Mol. Biol. Cell 22, 2290-2300 (2011).

92. Codogno, P., Mehrpour, M. \& Proikas-Cezanne, T. Canonical and non-canonical autophagy: variations on a common theme of self-eating? Nat. Rev. Mol. Cell Biol. 13, 7-12 (2011).

93. von Muhlinen, N. et al. LC3C, bound selectively by a noncanonical LIR motif in NDP52, is required for antibacterial autophagy. Mol. Cell 48, 329-342 (2012).

94. Florey, O., Kim, S. E., Sandoval, C. P., Haynes, C. M. \& Overholtzer, M. Autophagy machinery mediates macroendocytic processing and entotic cell death by targeting single membranes. Nat. Cell Biol. 13, 1335-1343 (2011).

95. Martinez, J. et al. Microtubule-associated protein 1 light chain 3 alpha (LC3)-associated phagocytosis is required for the efficient clearance of dead cells. Proc. Natl Acad. Sci. USA 108, 17396-17401 (2011). 


\section{Emerging regulation and functions of autophagy}

\section{Patricia Boya, Fulvio Reggiori and Patrice Codogno}

Nat. Cell Biol. 15, 713-720 (2013); corrected after print 1 July 2013

In the print version of this Review, Table 2 was mistakenly omitted. It appears correctly in the HTML and PDF versions. 